\title{
Seismo-ionospheric coupling correlation analysis of earthquakes in Greece, using empirical mode decomposition
}

\author{
G. S. Tsolis and T. D. Xenos \\ Aristotle University of Thessaloniki, Department of Electrical and Computer Engineering, 54006 Thessaloniki, Greece
}

Received: 17 November 2008 - Revised: 15 January 2009 - Accepted: 22 January 2009 - Published: 19 February 2009

\begin{abstract}
Ionospheric variability as a result of earthquake events is a confirmed phenomenon as published in various seismo-ionospheric coupling studies. Generally, ionospheric variations resulting from earthquake activity are much weaker than disturbances generated by different sources, e.g. geomagnetic storms. However, geomagnetic storm disturbances exhibit more global behaviour, whereas seismo-ionospheric variations occur only locally in an area that is specified by the magnitude of the earthquake. Crosscorrelation coefficient analysis is a technique proposed some years ago, and ensures cancelation of geomagnetic storm variations of the ionospheric plasma, provided that the measurements are taken from stations with similar behaviour in these phenomena. In this paper we will use the aforementioned technique for analyzing data from ionospheric stations in Rome and Athens, and apply it to a series of earthquakes in Greece. Considering the local behaviour of the seismoionospheric variations, we expect that the Athens station, which happens to be inside the area affected by the earthquake, will accurately capture the disturbances. Due to its distance from the activity, we also do not expect the Rome station measurements to be affected by the seismic events in Greece. In addition, due to the fact that ionospheric plasma parameters exhibit non-stationary and nonlinear behaviour, we propose a novel signal processing technique known as the Hilbert-Huang transform in order to denoise the data before we calculate the cross-correlation coefficient of the two signals. Results from our analysis are in accordance with previously-conducted studies covering the same topic, clearly demonstrating that there are ionospheric precursors 1 to 7 days prior to strong seismic events as well as 1 to 2 days following such events.
\end{abstract}

Correspondence to: G. S. Tsolis strantis@gmail.com

\section{Introduction}

Seismo-ionospheric coupling phenomena have been studied for almost half a century now. The ability to use this data to provide early, as well as valid, warning of upcoming earthquakes is still controversial. This is mostly due to the fact that the ionosphere is a very complex and nonlinear system, in addition to the fact that there are a large number of parameters contributing to its variability. This means that it is not always possible to define the exact source of an ionospheric disturbance, since this can be the final nonlinear result of many independent factors acting simultaneously. Therefore the real problem is distinguishing the variations caused by earthquake activity from those stimulated by other sources.

It is well known that ionospheric variations are strongly related to the 11 year solar cycle. Phenomena that affect ionospheric variability such as ionospheric storms, polar cap absorption (PCA), travelling ionospheric disturbances, and magnetic storms are associated, either directly or indirectly, with the activity of the sun. As a result, ionospheric parameters vary diurnally, from hour to hour and even in time increments of less than an hour. Moreover, there are fluctuations in the ionosphere electron density which cannot be attributed to any one of the aforementioned sources, and are thus known as geophysical noise (Davies, 1990).

Ionospheric variability is usually studied using ground ionosondes and by measuring the electron density of ionospheric plasma or the integral of the electron density, known as Total Electron Content (TEC) along a ray path from a satellite based ionosonde (Kouris et al., 2001). The parameter of interest when the ground ionosondes are used is the critical frequency of the F2-layer, foF2. Having already mentioned the chaotic and nonlinear behavior of the ionosphere, it is reasonable to assume that the foF 2 measurements over a given period form a signal which consists of linear, nonlinear and nonstationary components.

Published by Copernicus Publications on behalf of the European Geosciences Union and the American Geophysical Union. 


\section{Seismo-ionospheric coupling phenomena}

So far a significant number of large-scale earthquakes have been studied using the seismo-ionospheric coupling point of view, the first one being the Good Friday Alaska Earthquake which occurred on 27 March 1964 (Pulinets, 2004). Similar results contribute to the conclusion that ionosperic variations occur 1 to 7 days prior to an earthquake event (Liu et al., 2001). Meta-seismic perturbations were also evidenced in some cases up to 2 days after the event (Gokhberg et al., 1995).

The physical explanation of the phenomenon is that due to gas emanations from the earth's crust $\left(\mathrm{CO}_{2}\right.$, Radon, light gases, etc.) prior to an earthquake, neutral clusters of atmospheric plasma are destroyed, enriching the atmosphere with ions, and producing an anomalous vertical electric field that penetrates through the ionosphere (Pulinets et al., 1997; Pulinets, 2004; Molchanov et al., 2004). This anomalous electric field is not confined only to the earthquake epicenter, but occupies an area that is a function of the magnitude of the earthquake, and is known as the earthquake preparation zone. The radius of this zone $\rho$ is a function of the earthquake magnitude $M$, and is given by the following equation (Dobrovolsky et al., 1979).

$\rho=10^{0.43 M} \mathrm{~km}$

However, in order for the anomalous electric field to effectively penetrate into the ionosphere, the size of the earthquake preparation zone should be larger than $200 \mathrm{~km}$, which corresponds to an earthquake with a magnitude $M \geq 4.65$. To this end a threshold of $M=5$ can be used for a meaningful analysis of the seismo-ionospheric coupling, understanding that a buffer of 0.35 ensures precision of the resultant data (Pulinets, 2004).

\section{Correlation analysis}

The majority of the methods used in seismo-ionospheric coupling studies are statistical methods, where some ionospheric parameters (usually foF2 or TEC) are evaluated for a period both before and after the earthquake. The main objective in all of these studies is to reveal a significant deviation of the examined parameter from its median value prior to the seismic event. Frequently, in an effort to eliminate the diurnal variation of foF 2 or TEC, their normalized equivalents are used, that is, the difference between the foF2 (or TEC) value from its monthly median, divided by the monthly median. However, even though this technique ensures a more stable signal than the raw foF2 data, geophysical noise still exists in the signal, which may render the analysis problematic. Moreover, one should be very careful trying to detect earthquake precursors using foF2, (TEC) or even DfoF2, (DTEC) data, since variations caused by other sources such as ionospheric storms or geomagnetic perturbations, may contaminate the data and therefore make the precursors difficult to be localized, if not totally obscured.

Correlation analysis is a method which promises to offset this kind of problem, that is, the shadowing of seismoionospheric precursors by the effects of geomagnetic storms, since the latter produce more intense disturbances in the F2layer critical frequency than the earthquakes do (Pulinets, 2004). In this method the foF 2 data from two different stations are used for the same period of time both prior to and after an earthquake. The two stations must be carefully selected, however, in such a way that the first one is inside the earthquake preparation area, and the other outside of it.

The idea is that the geomagnetic or ionospheric storms have more global effects, whereas the seismo-ionospheric coupling variations are characterized by their local behavior in that they appear only inside the earthquake preparation area. Thus the behavior of the two stations is expected to be the same as with geomagnetic storms, provided of course that they are in the same (or very close) geomagnetic latitude, hence their correlation coefficient will be high. On the contrary, their correlation coefficient is expected to drop in the event of earthquake activity where the epicenter is closer to one of the two stations. In order for the technique to have more accurate results, the two stations should not differ too much in longitude, due to the Local Time dependence of the ionospheric variability caused by the magnetic storms $(\mathrm{Pu}-$ linets et al., 2004).

The technique has been tested with success on a series of earthquake events in the Western Pacific (Russia, Far East, Taiwan, and Japan). Promising results were also reported in the case of the 21 January Colima Earthquake in Mexico, as well as in the San Simeon Earthquake of December 2003 in California. In both cases TEC data were used, proving the applicability of this technique in various types of ionospheric data (Pulinets et al., 2004).

It should be mentioned however, that although correlation analysis ensures cancelation of the geomagnetic storm effects due to the cross correlation function involved, there are still ionospheric variations present in the two signals caused by unknown sources, which can be uncorrelated. Therefore, the cross correlation coefficient between the two signals may appear very low due to the existence of this geophysical noise even in the absence of seismo-ionospheric coupling activity. Removing the geophysical noise from the signals prior to conducting the cross correlation coefficient calculation, will improve the validity of the technique, and ultimately provide more accurate results.

\section{Hilbert-Huang Transform}

Traditional filtering methods are realized in the frequency domain only. However, filtering in the frequency domain is very difficult to effectively implement for nonstationary and nonlinear signals. This is mostly due to the fact that 
frequency analysis of nonlinear and nonstationary signals generates harmonics in a wide range, and as a result any filtering in the frequency domain will eliminate some of the harmonics, and eventually will cause deformation of the waveforms of the fundamental modes, if they are outside the filtering range.

The Hilbert-Huang Transform (HHT), is a data driven signal processing method proposed by Norden Huang of NASA in 1998. It consists of two parts, the first being the Empirical Mode Decomposition, where the signal is decomposed in a series of structural components, known as Intrinsic Mode Functions (IMF). An IMF is defined as any function having the same number of zero-crossings and extrema, and also having symmetric envelopes defined by the local maxima and minima, respectively (Huang et al., 1998, 2005). IMFs admit well-behaved Hilbert transforms, and thus the second part of the method is just the Hilbert transform, which provides instantaneous frequencies as a function of time for each one of the IMF components.

Compared to the legacy signal processing methods, namely Fourier transform or Fast Fourier Transform, as well as more contemporary ones, such as Wavelets analysis, HHT exhibits two significant advantages. First, it is highly applicable to non-linear and non-stationary signal processing, since it is based on the local characteristic time scale of the data. Secondly it is totally adaptive and data driven, due to the fact that there is no need for a-priori selection of a basis (i.e., mother wavelet) for the data analysis. For the interested reader a thorough analysis of the Hilbert-Huang transform is provided in the original paper submitted by Norden Huang in 1998 and also in Huang et al. (2005). Here we will attempt a brief description of the algorithm explaining the key components.

For a real signal $x(t)$ the EMD starts by defining the envelopes of its maxima and minima using cubic splines interpolation. Then the mean of the two envelopes is calculated as

$m_{1}(t)=\frac{E_{\max }(t)+E_{\min }(t)}{2}$

Accordingly, the mean $m_{1}(t)$ is then subtracted from the original signal

$h_{1}(t)=x(t)-m_{1}(t)$

and the residual $h_{1}(t)$ is examined as for the IMF criteria completeness. If it is an IMF then the procedure stops and the new signal under examination is the

$x_{1}(t)=x(t)-h_{1}(t)$

However, if $h_{1}(t)$ is not an IMF, the procedure, also known as "sifting", is continued $k$ times until the first IMF is realized. Thus,

$h_{11}(t)=h_{1}(t)-m_{11}(t)$ and finally

$\operatorname{IMF}_{1}=h_{1 k}(t)=h_{1(k-1)}(t)-m_{1 k}(t)$

The sifting process is continued until the last residual is either a monotonic function or a constant. It should be mentioned that as the sifting process evolves the number of the extrema from one residual to the next drops, thus guaranteeing that the complete decomposition is achieved in a finite number of steps. The final product is a wavelet-like decomposition going from higher oscillations to lower oscillations, which means that the frequency spectrum is decreased as the order of the IMF increases. The big difference, however, with the wavelet analysis is that while modes and residuals can intuitively be given a "spectral" interpretation in the general case, their high versus low frequency discrimination applies only locally and corresponds in no way to a predetermined subband filtering. Selection of modes instead corresponds to an automatic and adaptive (signal-dependent) time-variant filtering (Flandrin et al., 2003).

After completion of the EMD the signal can be written as follows,

$x(t)=\sum_{i=1}^{n} \mathrm{IMF}_{i}+r_{n}$

where $n$ is the total number of the IMF components and $r_{n}$ is the residual. The completeness of the method is being verified since the difference between the initial signal and the signal given by the Eq. (7), is less than $5 \times 10^{-15}$. Also performing the Hilbert transform in each one of the IMF components, excluding the final residual, the signal can be expressed as

$x(t)=\operatorname{real} \sum_{j=1}^{n} a_{j}(t) e^{i \int \omega_{j}(t) d t}$

where $a_{j}(t)$ is the amplitude of the IMF as a function of time. The above expression is clearly a generalized Fourier representation of the signal.

In Fig. 1 the analysis of a foF2 signal into its IMF components is presented for illustrative purposes.

\section{5 foF2 signal denoizing using Empirical Mode Decomposition}

The filtering process associated with the Hilbert-Huang transform is related to the reconstruction property of the signal by its IMF components. In fact, the EMD acts essentially as a dyadic filter bank, much like the wavelet decompositions, which means that the high oscillations are captured in the low order IMF components, whereas the low frequency waves are captured by higher order IMFs (Flandrin et al., 2003, 2004). To this end reconstructing the signal by only those components that contain the signal energy, and leaving out the noise which is captured in higher order IMF, provides 

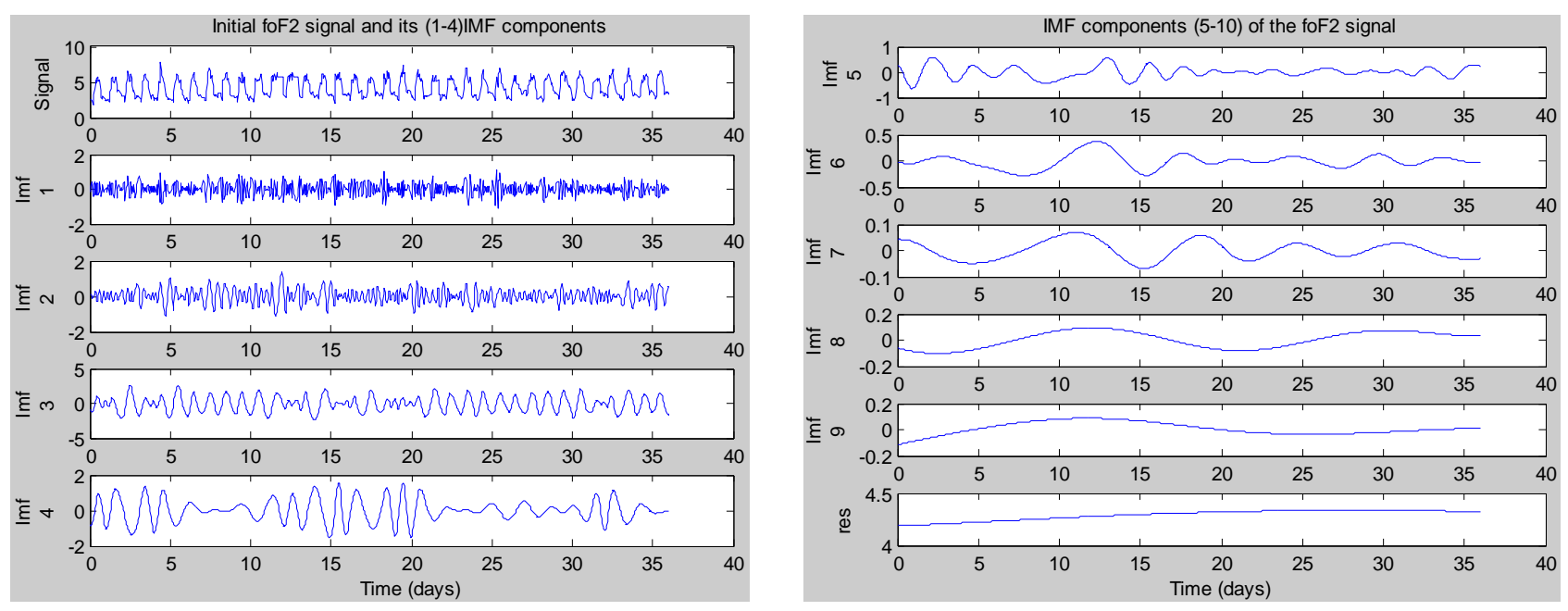

Fig. 1. foF2 signal from the Athens station, and its analysis in IMF components using the Hilbert-Huang transform.
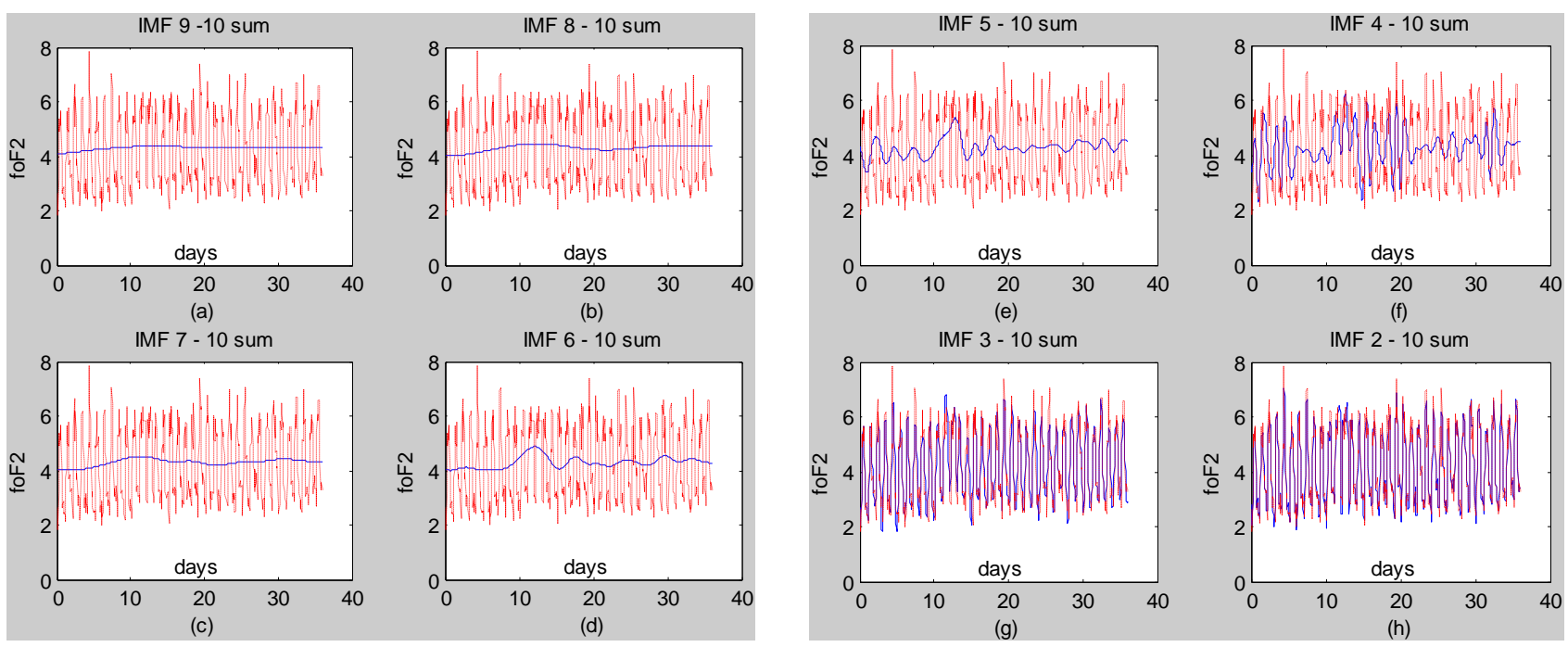

Fig. 2. Reconstruction of the foF2 signal adding its IMF components. From (a) through (h), one IMF component is added each time, until the initial signal is reconstructed.

a noise-free equivalent of the initial data. We demonstrate the procedure in Fig. 2 using a foF2 signal from the Athens Ionosonde station. Starting from $2 \mathrm{a}$ and proceeding up to $2 \mathrm{~h}$, the initial signal is represented by the dotted line, and the sum of the IMFs is represented by the solid line.

The question arises as to how many of the low order IMF must be excluded from the reconstruction in order for the noise to be left out of the signal. In our case the number of IMFs that are excluded from the reconstruction is a result of an a-priori decision based upon the physical characteristics of the signal. In fact we have excluded the first two IMFs since they exhibit noisy behaviour. The reconstructed signal, which is a result of the summation of the remaining IMF components, is in accordance with the diurnal variation of the foF 2 . This is clearly illustrated in Fig. 3 where the foF2 signal is presented together with the denoised version.

\section{Data analysis}

In this study we used foF2 data from the Athens (Lat. 38.0Lon. 23.5) and Rome (Lat. 41.8-Lon. 12.5) ionospheric stations, made available by Geophysical Laboratory of the Aristotelles University of Thessaloniki Greece database.The foF2 data are $1 \mathrm{~h}$ sample recordings, and refer to a series of eight earthquakes in Greece, with magnitudes of 5.9 to 6.5, during the 5 year period from 2003-2008. Unfortunately it was not possible to study all of the earthquakes recorded during this specific period of time due to the absence of sufficient data.

In all earthquakes studied, Athens station was inside the earthquake preparation area - except for the 17 March event where it was slightly outside ( $25 \mathrm{~km})$ - as illustrated in Fig. 4. Rome station was outside of the affected area for all earthquakes. Therefore, Athens recordings are expected to depict 

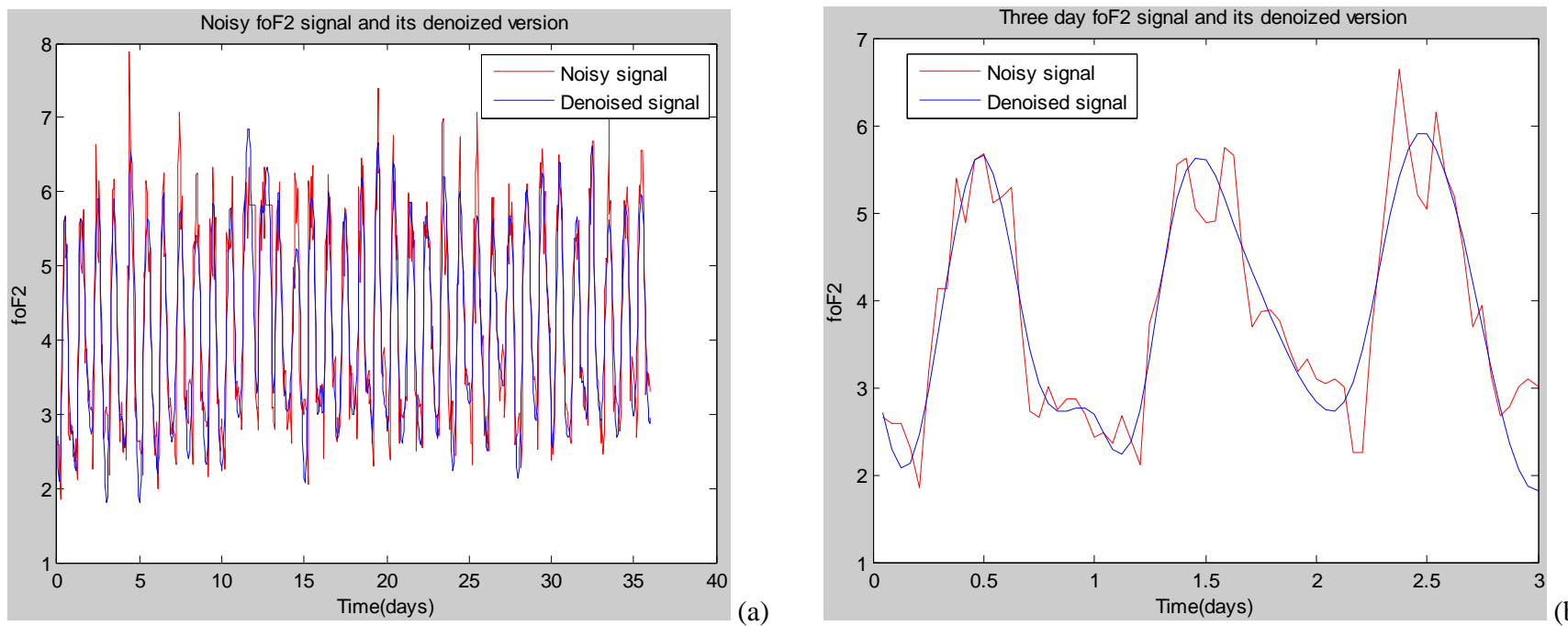

Fig. 3. Initial foF2 signal (red line) and its de-noised version (blue line), using the Hilbert-Huang transform. (a) 36 day signal, (b) 3 day signal.

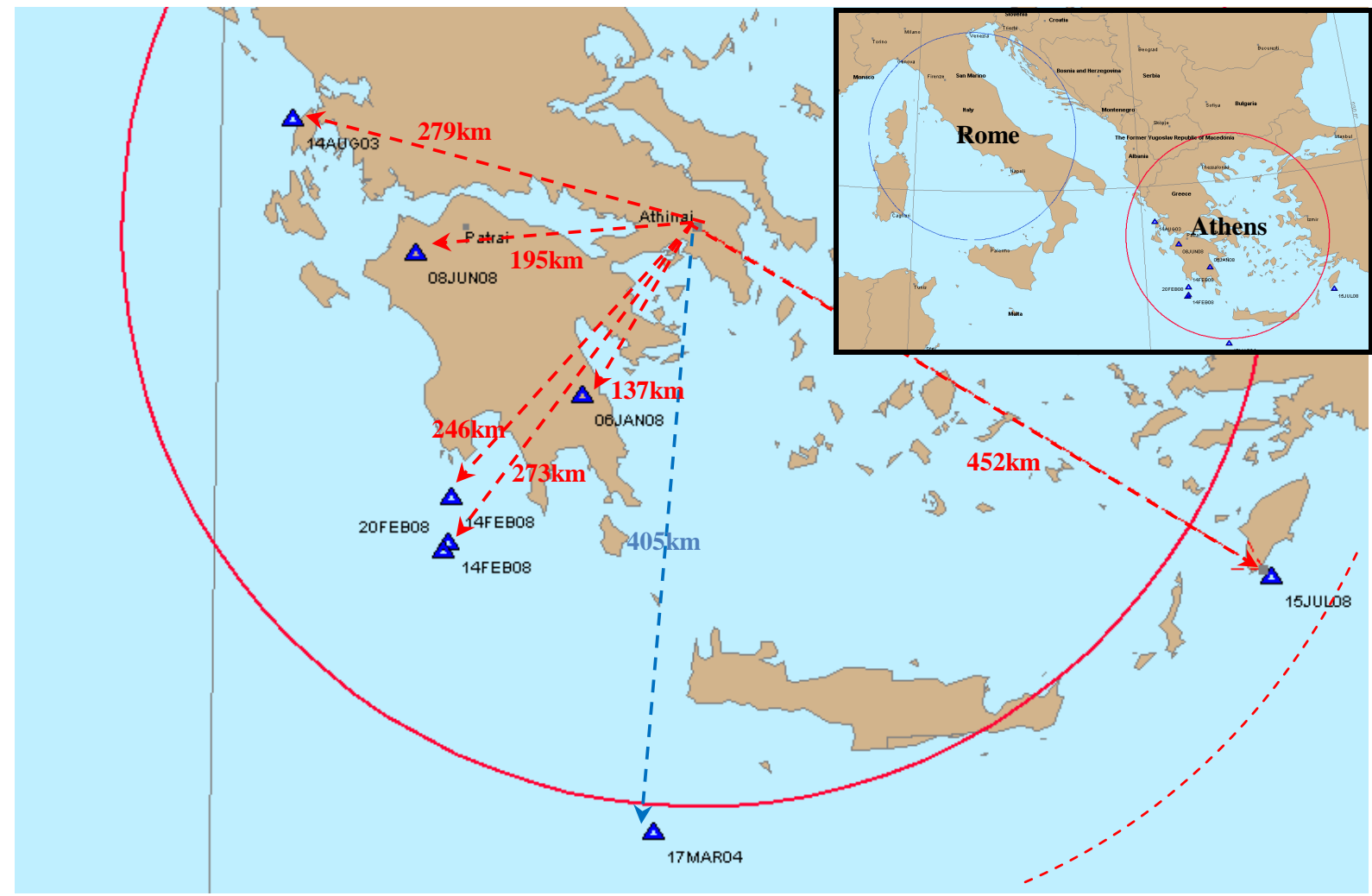

Fig. 4. The distances of the earthquake epicenters from the Athens station. Red circle indicates an earthquake preparation area which corresponds to an event of $M_{w}=6(380 \mathrm{~km})$. For all the earthquakes the Athens station was inside the earthquake preparation area (red dotted arrows), except for the event of 17 March 2004, where the Athens station was just outside of the earthquake preparation area (blue dotted line). For the 15 July earthquake the earthquake preparation area was $463 \mathrm{~km}$ (red dotted circle).

ionospheric variation due to seismic activity. On the contrary, the Rome recordings are expected to be insensitive to the ionospheric variation due to seismic events. On the other hand, foF 2 variations caused by the geomagnetic storms are expected to be the same, since the LT difference of the two stations is very small (only $1 \mathrm{~h}$ ), and the geomagnetic latitude of the two stations differs only slightly. 

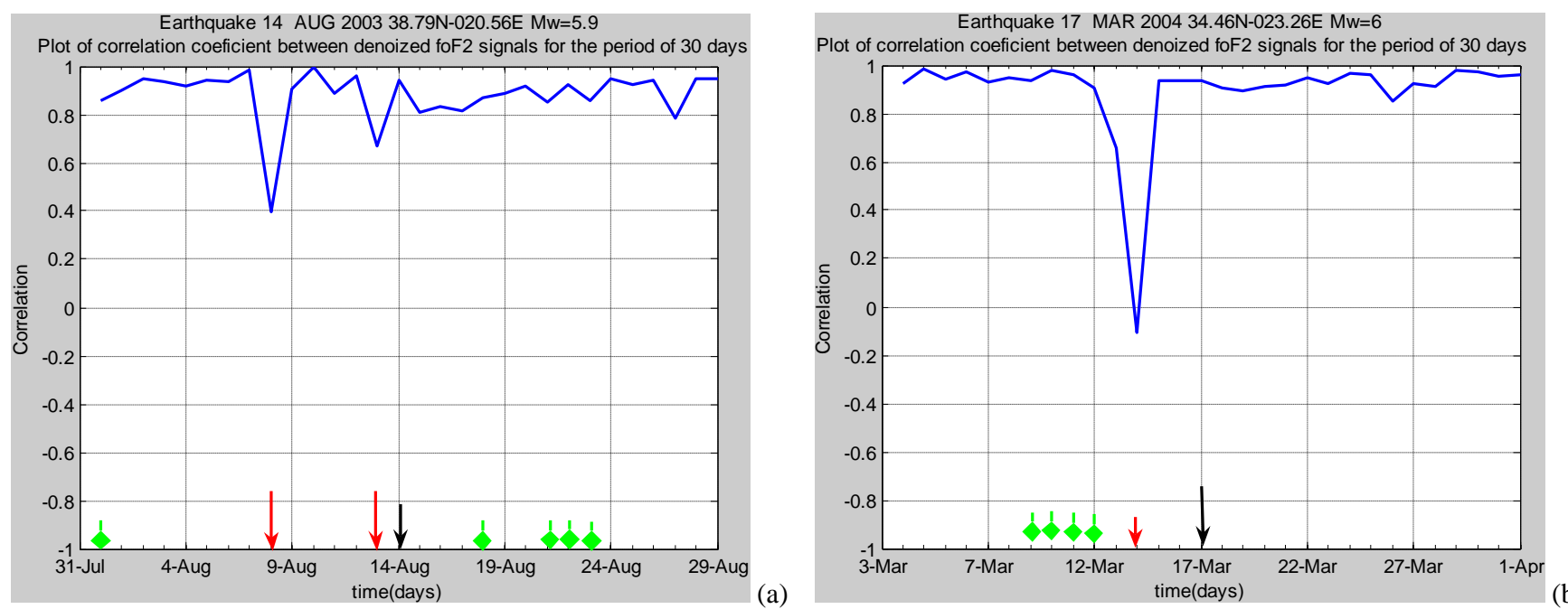

Fig. 5. Plot of the correlation coefficient of the denoised foF 2 signals from the Athens and Rome ionosonde stations for 14 August 2003 and 17 March 2004 earthquakes. Black arrows indicate days of the seismic events, red arrows represent ionospheric precursors and green diamonds indicate magnetically disturbed days.

For every earthquake a 15 day period prior and after the event was studied. This period was shorter (12 days) for some of the events, due to the non-availability of data. The foF 2 signals were initially processed using the HilbertHuang transform and the denoised version was produced. Then for each earthquake the cross correlation between the denoised versions of the Athens and Rome recordings was calculated.

\section{Results and discussion}

\subsection{Earthquake 14 August 2003}

The epicentre of the earthquake was located at $38.79 \mathrm{~N}-$ $020.56 \mathrm{E}$. The magnitude of the event was 5.9 and the depth $12 \mathrm{~km}$. The distance from Athens station was $279 \mathrm{~km}$, and the distance from Rome station was $810 \mathrm{~km}$. Using Eq. (1), the earthquake preparation area is calculated as $344 \mathrm{~km}$, and therefore Athens is expected to pickup the ionospheric precursors. In Fig. 5a the cross-correlation coefficient between the denoized foF2 signals is plotted for the period of 30 days, from 31 July to 29 August 2003. As shown the crosscorrelation coefficient initially drops 5 days prior to the earthquake on 9 August, and also 1 day before on 14 August.

\subsection{Earthquake 17 March 2004}

The event occurred $405 \mathrm{~km}$ south of Athens at $34.46 \mathrm{~N}-$ $023.26 \mathrm{E}$, in a depth of $24 \mathrm{~km}$, and its magnitude was 6.0 . Here the correlation coefficient is calculated for the denoised foF2 signals from 3 March to 1 April 2004. Although Athens station is just outside the earthquake preparation area, as shown in Fig. 5b there is a severe drop in the cross correlation coefficient 3 days before the earthquake, on 14 March 2004.

\subsection{Earthquake 6 January 2008}

The epicentre of the earthquake was located $137 \mathrm{~km} \mathrm{SW}$ from Athens, at $37.11 \mathrm{~N}-022.78 \mathrm{E}$, and the magnitude was 6.1 . The correlation coefficient of the de-noised foF 2 signals for a 30 day period (23 December 2007-22 January 2008) is plotted in Fig. 6a. Note that despite the fact that the depth of the event was considerably deep $(86 \mathrm{~km})$, the cross-correlation coefficient drops 7 and 6 days before the event on 30 and 31 December, respectively.

\subsection{Earthquakes on 14 and 20 February 2008}

On 14 and 20 February 2008 three earthquake evens occurred in the Greek territory. As shown in Fig. 7 the 14 February earthquakes recorded very close to each other $273 \mathrm{~km} \mathrm{SW}$ from Athens, at coordinates $36.5 \mathrm{~N}-021.78 \mathrm{E}$ and $36.22 \mathrm{~N}-$ $021.75 \mathrm{E}$, respectively. Their magnitude was 6.2 and 6.1, and they occurred in a depth of $41 \mathrm{~km}$ and $38 \mathrm{~km}$, respectively. The third earthquake occurred also in the vicinity of the previous two $246 \mathrm{~km} \mathrm{SW}$ from Athens, at $36.18 \mathrm{~N}-021.72 \mathrm{E}$, in a depth of $25 \mathrm{~km}$ and its magnitude was 6 . For these three events the correlation coefficient was calculated using the denoised foF 2 signals, for the period 15 days before the first and 15 after the last event, that is, 36 days in total. As shown in Fig. 6b there is a drop in the correlation coefficient on 13 February, which corresponds to a precursor 1 day before the 14 February earthquakes and 7 days before the 20 February event. Also there is another even more intense drop on 19 February, 1 day before the 20 February earthquake.

\subsection{Earthquake on 8 June 2008}

The epicentre of the event was located $195 \mathrm{~km} \mathrm{~W}$ from Athens, at $37.98 \mathrm{~N}-021.51 \mathrm{E}$, in a depth of $25 \mathrm{~km}$. It was 

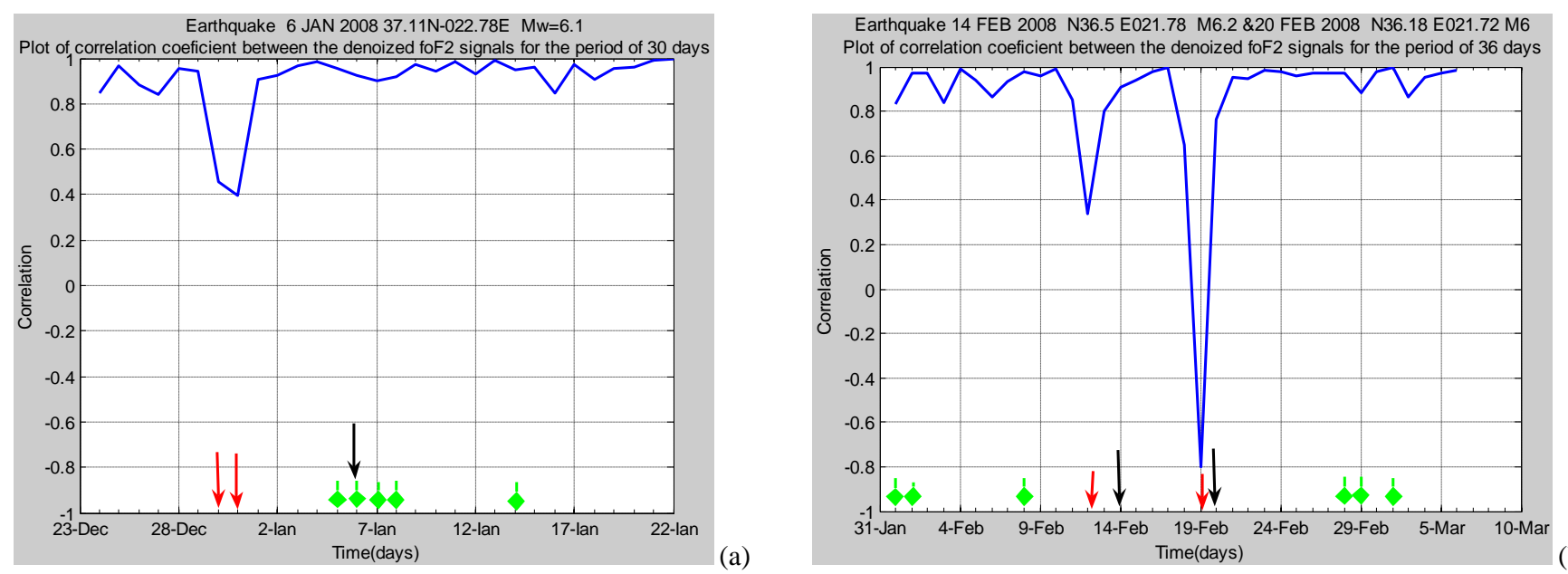

Fig. 6. Plot of the correlation coefficient of the denoised foF 2 signals from the Athens and Rome ionosonde stations for the 6 January 2008 , 14 February 2008 and 20 February 2008 earthquakes. Black arrows indicate days of the seismic events, red arrows represent ionospheric precursors and green diamonds indicate magnetically disturbed days.
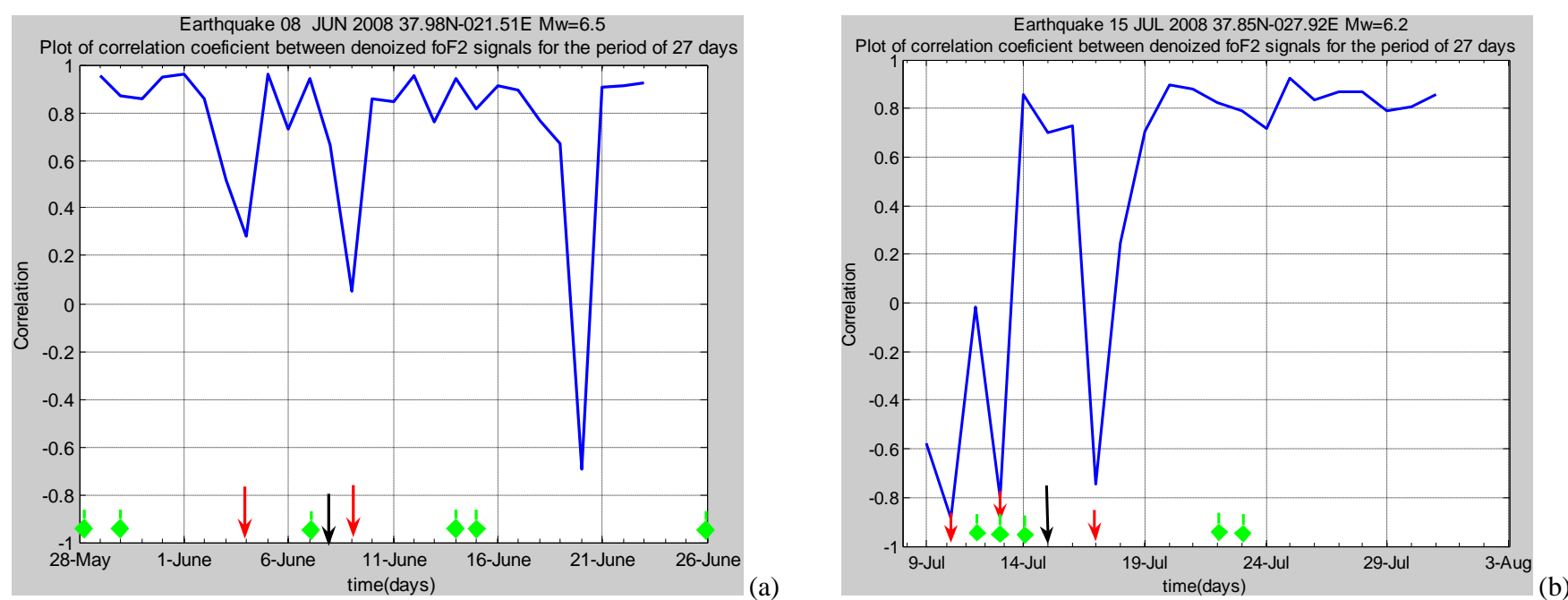

Fig. 7. Plot of the correlation coefficient of the denoised foF 2 signals from the Athens and Rome ionosonde stations for the 8 June 2008 and 15 July 2008 earthquakes. Black arrows indicate days of the seismic events, red arrows represent ionospheric precursors and green diamonds indicate magnetically disturbed days. Correlation coefficient drop on 21 June cannot be justified as ionospheric precursor.

a strong 6.5 M seismic event, and since it occurred over land, resulted in the loss of human lives as well as significant damages to buildings. In Fig. 7a the correlation coefficient of the denoised foF 2 signals is plotted for the 27 day period from 28 May to 26 June 2008. This corresponds to 15 days after the event and only 12 days ahead of it, since for the days before the 28 of May the data was insufficient. In this case there are correlation coefficient drops 4 days before the event on 5 June, and 1 day after the earthquake on 9 June. These low correlation coefficient values can be accepted as earthquake precursors. However, there is a severe drop of the coefficient 12 days after the event, which is also placed 17 days prior to the earthquake on 15 July 2008, but cannot be justified as a precursor based upon the current theory and experimental results of previous seismo-ionospheric coupling studies.

\subsection{Earthquake on 15 July 2008}

The earthquake occurred $452 \mathrm{~km}$ SE from Athens at $35.85 \mathrm{~N}-027.92 \mathrm{E}$ in a depth of $56 \mathrm{~km}$, and its magnitude was 6.2. The correlation coefficient of the denoised foF2 signals is calculated for a period of 25 days since the data before 8 July 2008 was insufficient. In Fig. 7b precursors are shown 4 and 2 days before the event on 11 and 13 July, respectively, as well as 2 days after it, on 17 July. 


\section{Conclusions}

In this paper we applied the Cross-Correlation Coefficient method, combined with the Hilbert-Huang Transform, for the analysis of ionospheric data in a series of earthquakes in Greece. The main advantage of the Cross-Correlation Coefficient method is that it ensures cancelation of disturbances in the ionosphere caused by geomagnetic storms, and thus much weaker variations caused by earthquake events can be revealed. For this we use data for two different stations, both inside and outside of the earthquake preparation area namely Athens and Rome, respectively. In addition, since ionospheric variability is a result of various parameters, including geophysical noise, we treated the foF2 measurements collected by Rome and Athens ionosonde stations as non-stationary and nonlinear signals, and we denoised using the data driven and adaptive method of the Hilbert-Huang transform. The results are very promising confirming the existence of ionospheric precursors before strong earthquake events. Specifically we found that the correlation coefficient of the denoised foF2 signals drops 7 to 1 days before the earthquake and 1 to 2 days after the event, which is in accordance with the results of previous seismo-ionospheric studies. In our study we also experienced a single case where an ionospheric precursor was recorded without being followed by a seismic event, which was the case with the correlation coefficient drop of 21 June of 2008. Although there was actually an earthquake 17 days after this precursor, we cannot conclude that this correlation coefficient drop was related to this event, since a case like this was never reported in similar studies. A possible explanation for this can be some data inaccuracy in one of the stations we used as a source for the ionospheric data. In any event the existent theoretical and experimental knowledge is insufficient to justify this correlation coefficient drop as an ionospheric precursor, hence we leave it as a subject for our future studies.

Edited by: J. Kurths

Reviewed by: one anonymous referee

\section{References}

Davies, K.: Ionospheric Radio, Peregrinus Ltd., London, UK, 1990.

Dobrovolsky, I. R., Zubkov, S. I., and Myachkin, V. I.: Estimation of the size of earthquake preparation zones, Pageoph., 117, 1025-1044, 1979.

Flandrin, P., Rilling, G., and Gonçalvès, P.: Empirical Mode Decomposition as a filter bank, IEEE Signal Proc. Lett., 2003.

Flandrin, P., Gonçalvès, P., and Rilling, G.: Detrending and denoising with Empirical Mode Decompositions, Eusipco, 12th European Signal Processing Conference, 6-10 September, Vienna, Austria, 2004.

Gokhberg, M. B., Morgounov, V. A., and Pokhotelov, O. A.: Earthquake Prediction, Seismo-electromagnetic phenomena, Gordon and Breach Science Publishers, Amsterdam, 1995.

Huang, N. E., Shen, Z., Long, S. R., Wu, M. L., Shih, H. H., Zheng, Q., Yen, N. C., Tung, C. C., and Liu, H. H.: The empirical mode decomposition and Hilbert spectrum for nonlinear and nonstationary time series analysis, P. Roy. Soc. Lond. A, 454, 903-995, 1998.

Kouris, S. S., Spalla, P., and Zolesi, B.: Could ionospheric variations be precursors of a seismic event? A short discussion, Annals of Geophysics, Series/Report N44/2, 2001.

Liu, J. Y., Chen, Y. I., Chuo, Y. J., and Tsai, H. F.: Variations of ionospheric total content during the Chi-Chi earthquake, Geophys. Res. Lett., 28, 1381-1386, 2001.

Molchanov, O., Fedorov, E., Schekotov, A., Gordeev, E., Chebrov, V., Surkov, V., Rozhnoi, A., Andreevsky, S., Iudin, D., Yunga, S., Lutikov, A., Hayakawa, M., and Biagi, P. F.: Lithosphereatmosphere-ionosphere coupling as governing mechanism for preseismic short-term events in atmosphere and ionosphere, Nat. Hazards Earth Syst. Sci., 4, 757-767, 2004, http://www.nat-hazards-earth-syst-sci.net/4/757/2004/.

Pulinets, S. A., Alekseev, V. A., Legenka, A. D., and Hegai, V. V.: Radon and Metallic Aerosols Emanation before Strong Earthquakes and their Role in Atmosphere and Ionosphere Modification, Adv. Space Res., 20, N11, 2173-2176, 1997.

Pulinets, S.: Ionospheric precursors of earthquakes; Recent advances in theory and practical applications, TAO, 15(3), 413435, 2004.

Pulinets, S. A., Gaivoronska, T. B., Leyva Contreras, A., and Ciraolo, L.: Correlation analysis technique revealing ionospheric precursors of earthquakes, Nat. Hazards Earth Syst. Sci., 4, 697702, 2004,

http://www.nat-hazards-earth-syst-sci.net/4/697/2004/. 